\title{
Hyperhomocysteinaemia predicts the decline in pulmonary function in healthy male smokers
}

\author{
Keiko Nunomiya', Yoko Shibata1, Shuichi Abe1, Sumito Inoue1, Akira Igarashi', \\ Keiko Yamauchi', Yasuko Aida', Hiroyuki Kishi', Masamichi Sato', \\ Tetsu Watanabe', Tsuneo Konta', Yoshiyuki Ueno², Takeo Kato², \\ Hidetoshi Yamashita², Takamasa Kayama² and Isao Kubota'
}

Affiliations: 'Dept of Cardiology, Pulmonology and Nephrology, Yamagata University School of Medicine, Yamagata, and ${ }^{2}$ Global Centre of Excellence Programme Study Group, Yamagata University School of Medicine, Yamagata, Japan.

Correspondence: Y. Shibata, Dept of Cardiology, Pulmonology and Nephrology, Yamagata University School of Medicine, 2-2-2 lida-Nishi Yamagata 990-9585, Japan. E-mail: shibatalamed.id.yamagata-u.ac.jp

ABSTRACT Hyperhomocysteinaemia is associated with chronic obstructive pulmonary disease. However, the relationship between plasma homocysteine levels and spirometric measures has not been investigated in a general population. We aimed to determine whether homocysteine levels are predictive for a rapid decline in lung function among healthy current smokers.

Blood sampling and spirometry were performed on subjects participating in a community-based annual health check in Takahata, Japan, from 2004 to $2006(n=3257)$. Spirometry was re-evaluated in 147 male current smokers in 2009.

On initial assessment, forced vital capacity (FVC) \% predicted and forced expiratory volume in $1 \mathrm{~s}$ (FEV1) \% predicted correlated inversely with homocysteine levels and were predictive for homocysteine levels, independent of various clinical factors. Homocysteine levels were higher in subjects with restrictive, obstructive or mixed ventilatory disorders. In addition, homocysteine levels were higher in subjects with mixed ventilatory disorders, compared with restrictive or obstructive disorders. On follow-up, subjects showing a decline in FEV1 had higher homocysteine levels than those who did not. Logistic regression analysis indicated that homocysteine levels were predictive for a decline in FEV1.

FVC \% pred and FEV1 \% pred were significantly associated with homocysteine levels, and hyperhomocysteinaemia predicted the annual rate of decline in FEV1 among male smokers.

@ERSpublications

Plasma homocysteine levels predict rate of lung function decline in male smokers http://ow.ly/kEGBX

This article has supplementary material available from www.erj.ersjournals.com

Received: April 242012 | Accepted after revision: Oct 212012 | First published online: Nov 082012

Support statement: This study was supported by a grant-in-aid from the Global Centre of Excellence programme of the Japan Society for the Promotion of Science (F03).

Conflict of interest: None declared.

Copyright @ERS 2013 


\section{Introduction}

Long-term cigarette smoking injures the respiratory system and causes disorders such as chronic obstructive pulmonary disease (COPD) [1]. Even in healthy individuals, smoking causes a decline in pulmonary function [2]. In addition, patients with COPD have various comorbidities, including atherosclerosis, cachexia, diabetes mellitus and osteoporosis [3-8]. These comorbidities are thought to be associated with systemic inflammation due to the leakage of mediators from local inflammatory sites in the lung, and this is an important determinant of the morbidity associated with the disease [9].

Homocysteine plays an important role in the development of atherosclerosis [10-12] and is suggested to be involved in the pathogenesis of COPD [13-15]. Plasma homocysteine levels were found to be elevated in COPD patients and this is thought to be related to the stage of COPD [15]. However, homocysteine levels are influenced by other factors, such as sex [16], body mass index (BMI) [17], cigarette smoke inhalation $[18,19]$, blood pressure (BP) [20], diabetic nephropathy [21], hyperlipidaemia [22], liver function [23] and renal function (serum creatinine levels) [24]. Therefore, findings in relation to homocysteine levels need to be carefully assessed, with consideration of variation in the backgrounds of the subjects. To date, the relationship between homocysteine levels and spirometric measures has not been investigated in a general population. Furthermore, it remains to be elucidated whether homocysteine levels are predictive for a rapid decline in lung function among current smokers.

In this cross-sectional study, the relationship between spirometric parameters and homocysteine levels was investigated in participants in an annual health check in Takahata, Japan. Follow-up spirometric measurements were performed in 147 male current smokers, and the relationship between a decline in lung function and homocysteine levels was assessed.

\section{Methods \\ Study population}

This study was part of the Takahata Study, based on an annual community health check in which residents aged $\geqslant 40$ years participated. The study was approved by the institutional ethics committee and all participants gave written informed consent [2]. Cross-sectional analyses of spirometry data for subjects who were enrolled from 2004 to $2005(n=3165)$ have been reported previously [2, 25-27]. Enrolment of subjects continued through 2006, and the additional data for these subjects became available recently. 3520 subjects (1579 males and 1941 females) were enrolled. 263 subjects were excluded from the analysis because their spirometry data did not meet the specified criteria. The data for 3257 subjects (1502 males and 1755 females) were entered into the final statistical analysis. Subjects used a self-report questionnaire to document their smoking habits. The lifetime consumption of cigarettes was expressed as the Brinkman index (number of cigarettes per day $\times$ years of smoking). Of the 523 male current smokers, 147 performed subsequent follow-up spirometry in 2009.

\section{Measurements}

Systolic and diastolic BP were measured using a mercury sphygmomanometer. Mean BP was calculated as diastolic $\mathrm{BP}+0.33 \times($ systolic $\mathrm{BP}-$ diastolic $\mathrm{BP})$ [25]. Blood samples were taken from the antecubital vein of subjects who had been fasting.

Total plasma homocysteine concentrations were measured using an enzymatic homocysteine assay kit (MBL, Nagoya, Japan) [28].

Spirometric parameters (forced vital capacity (FVC) and forced expiratory volume in $1 \mathrm{~s}(\mathrm{FEV} 1)$ ) were measured using standard techniques, with subjects performing FVC manoeuvres on a CHESTAC-25 part II EX instrument (Chest Corporation, Tokyo, Japan), according to the guidelines of the Japanese Respiratory Society [29]. A bronchodilator was not administered prior to spirometry. The highest value from at least three FVC manoeuvres from each subject was used for the analysis. The rate of decline in spirometric measures $(\triangle \mathrm{FEV} 1$ per year $(\%)$ and $\triangle \mathrm{FVC}$ per year $(\%))$ were calculated as ([value at second spirometry value at first spirometry]/value at first spirometry) $\times 100 /$ time between observations (years).

\section{Statistical analysis}

For continuous variables, data are presented as mean $\pm \mathrm{SD}$ or median (interquartile range (IQR)). The t-test was used for parametric data and the Mann-Whitney U-test was used for nonparametric data to analyse the differences between two groups. For multiple comparisons, one-way ANOVA was used, followed by the Student-Newman-Keuls test. Correlations between two variables were evaluated using Pearson's product moment correlation coefficient. Univariate regression analysis was used to examine the association between homocysteine levels and each of the spirometric measures considered in this study. Multiple linear regression analysis was then performed to determine whether each of these spirometric measures 
contributed to homocysteine levels after adjustment for all other variables included in the model. A stepwise variable selection method was used to retain variables that reached a 0.20 level of significance. Results of multiple logistic regression analysis were presented as odds ratio and $95 \%$ confidence interval. Statistical significance was inferred for two-sided $p$-values $<0.05$. All statistical analyses were performed using JMP version 8 software (SAS Institute Inc., Cary, NC, USA).

\section{Results}

Assessment of results from the Takahata Study, 2004-2006

The characteristics of the subjects who were enrolled from 2004 to 2006 are summarised in table 1. In this study population, $64.4 \%$ of the subjects were never-smokers, and the Brinkman index did not show a normal distribution (median (IQR) 0 (0-175.5)). The distribution of homocysteine measurements was skewed towards higher values (median (IQR) 10.3 (8.7-12.3)). Therefore, homocysteine values were logarithmically transformed before analysis. Age, Brinkman index score, mean BP and alanine aminotransferase (ALT), serum creatinine, triglyceride and log homocysteine values were significantly higher in male subjects compared with female subjects. In contrast, total cholesterol levels, FVC \% pred and FEV1 \% pred were significantly lower in males than in females. BMI and haemoglobin ( $\mathrm{Hb}) \mathrm{Alc}$ levels did not differ significantly between males and females.

Figure 1 shows the relationship between spirometric parameters (FVC and FEV1) and log homocysteine values. There were inverse relationships between these spirometric parameters and log homocysteine levels.

Univariate regression analysis demonstrated that age, sex, Brinkman index, mean BP, ALT, serum creatinine, triglyceride, total cholesterol, FVC \% pred and FEV1 \% pred were significantly associated with $\log$ homocysteine levels (online supplementary table S1). Multiple linear regression analysis was performed to determine whether these spirometric measures contributed independently to homocysteine levels (table 2). As FVC \% pred and FEV1 \% pred were strongly correlated $(\mathrm{r}=0.85 ; \mathrm{p}<0.0001)$, these parameters were assessed separately to avoid confounding (table 2). As shown in table 2, FVC \% pred and FEV1 \% pred were predictive for homocysteine levels, independent of the other factors.

The possible relationship between increased homocysteine levels and impaired pulmonary function was investigated. The subjects were categorised according to their spirometry data, as follows. Normal spirometry: $\mathrm{FVC} \geqslant 80 \%$ pred and $\mathrm{FEV} 1 / \mathrm{FVC} \geqslant 0.7$; pure restriction: $\mathrm{FVC}<80 \%$ pred and $\mathrm{FEV} 1 / \mathrm{FVC} \geqslant 0.7$; pure obstruction: $\mathrm{FVC} \geqslant 80 \%$ pred and $\mathrm{FEV} 1 / \mathrm{FVC}<0.7$; and mixed ventilatory disorder: $\mathrm{FVC}<80 \%$ pred and FEV1/FVC $<0.7$ (fig. 2). Subjects with abnormal spirometry (restriction, obstruction or mixed disorders) had higher homocysteine levels than subjects with normal spirometry (fig. 2). Furthermore, subjects with a mixed

TABLE 1 Characteristics of study subjects

\begin{tabular}{|c|c|c|}
\hline & Males & Females \\
\hline Subjects & 1502 & 1755 \\
\hline Age years & $62.9 \pm 10.4$ & $61.8 \pm 10.3^{*}$ \\
\hline $\mathrm{BMI} \mathrm{kg} \cdot \mathrm{m}^{-2}$ & $23.5 \pm 3.0$ & $23.5 \pm 3.4$ \\
\hline Brinkman index cigarette-years & $441.0 \pm 495.6$ & $17.2 \pm 89.7^{\#}$ \\
\hline Mean BP $\mathrm{mmHg}$ & $99.8 \pm 11.0$ & $95.8 \pm 10.9^{\#}$ \\
\hline HbA1C \% & $5.26 \pm 0.75$ & $5.26 \pm 0.65$ \\
\hline ALT U. $L^{-1}$ & $25.9 \pm 15.4$ & $21.3 \pm 12.1^{* * *}$ \\
\hline $\mathrm{sCr} \mathbf{m g} \cdot \mathrm{dL}^{-1}$ & $0.78 \pm 0.27$ & $0.59 \pm 0.11^{\#}$ \\
\hline Triglycerides $\mathrm{mg} \cdot \mathrm{dL}^{-1}$ & $117.7 \pm 79.6$ & $98.7 \pm 47.3^{\#}$ \\
\hline Total cholesterol $\mathrm{mg} \cdot \mathrm{dL}^{-1}$ & $193.6 \pm 31.4$ & $206.8 \pm 31.4^{\#}$ \\
\hline Homocysteine $\mu \mathrm{M}$ & $12.6 \pm 6.9$ & $10.0 \pm 3.7^{\#}$ \\
\hline Log homocysteine & $1.08 \pm 0.13$ & $0.98 \pm 0.12^{\#}$ \\
\hline FVC $\%$ pred & $97.3 \pm 14.9$ & $99.8 \pm 14.3^{\#}$ \\
\hline FEV $1 \%$ pred & $95.6 \pm 17.5$ & $99.7 \pm 15.4^{\#}$ \\
\hline
\end{tabular}

Data are presented as $n$ or mean \pm SD. Brinkman index values were not calculated for 267 male subjects and 63 female subjects due to the lack of the information on "cigarette years" or "daily consumption of cigarettes". Data on homocysteine levels were not available for 29 male subjects and 94 female subjects. Differences between males and females were evaluated by t-test, except for Brinkman index and homocysteine levels where the Mann-Whitney U-test was used. BMI: body mass index; BP: blood pressure; Hb: haemoglobin; ALT: alanine aminotransferase; sCr: serum creatinine; FVC: forced vital capacity; \% pred: \% predicted; FEV1: forced expiratory volume in $1 \mathrm{~s} .{ }^{*}: \mathrm{p}<0.05 ;{ }^{* * *}: \mathrm{p}<0.001{ }^{*}: \mathrm{p}<0.0001$ compared with male subjects. 


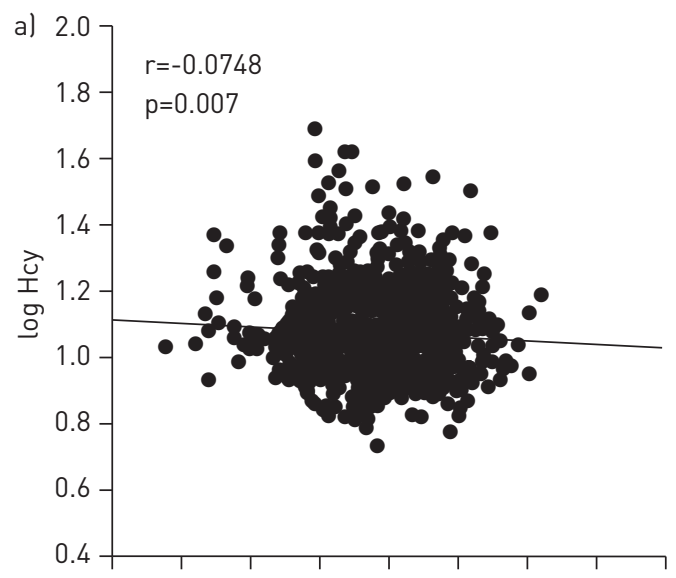

b)
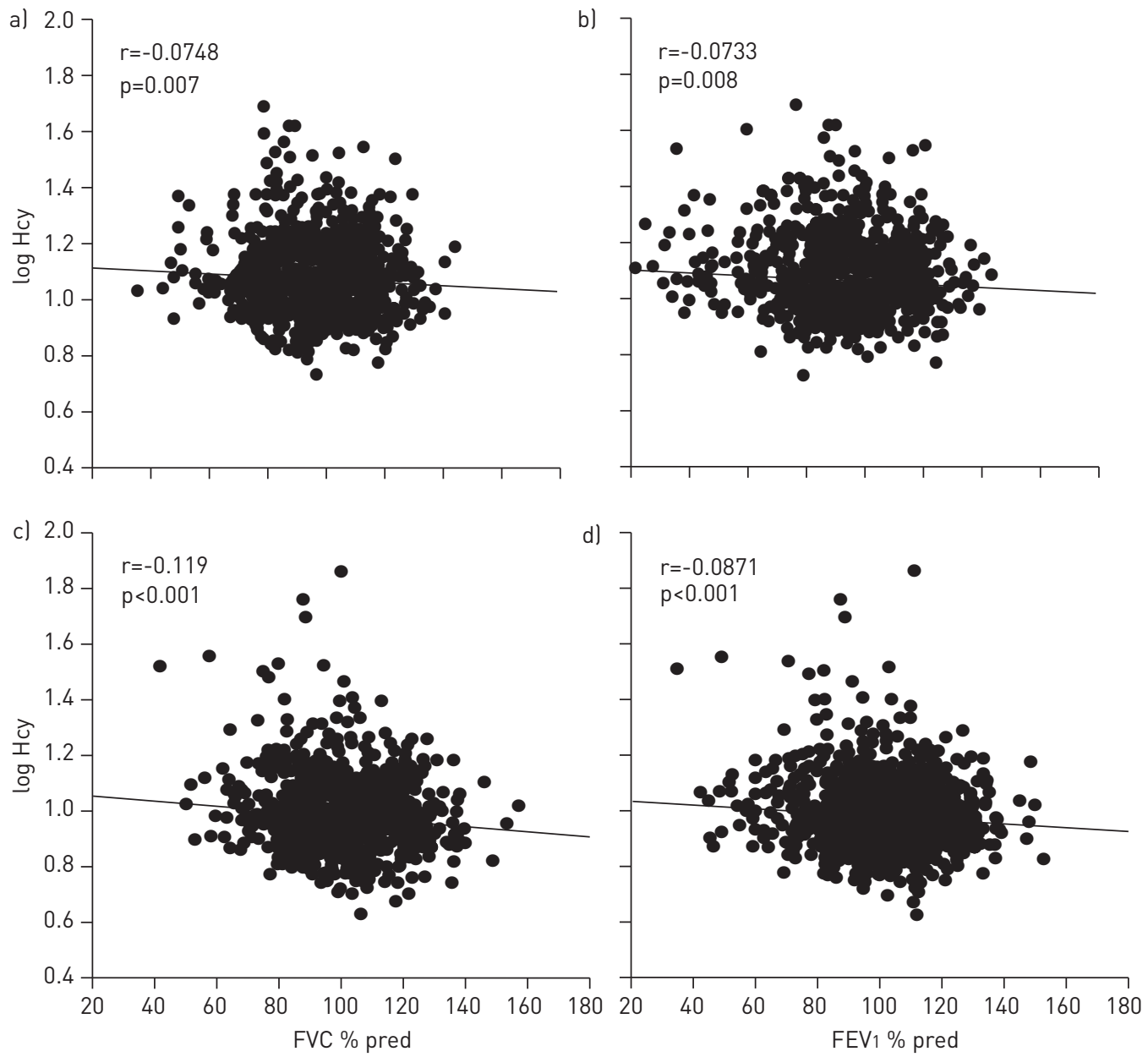

FIGURE 1 Inverse correlations between spirometric measurements and plasma homocysteine levels. Graphs show the relationships between the spirometric parameters $a, c)$ forced vital capacity (FVC) and b, d) forced expiratory volume in $1 \mathrm{~s}$ (FEV1) and plasma homocysteine (Hcy) levels in a, b) males and c, d) females. Correlations between spirometric measurements and log Hcy values were evaluated using Pearson's product moment correlation coefficient. There were inverse relationships between these spirometric parameters and plasma Hcy levels. \% pred: \% predicted.

ventilatory disorder had significantly higher homocysteine levels than subjects with either a restrictive or an obstructive disorder.

\section{Association between longitudinal changes in pulmonary function and plasma homocysteine levels in the Takahata study}

Of the 523 male current smokers who participated in the study, 147 performed follow-up spirometry in 2009 (characteristics of these subjects are summarised in table 3). BMI, Brinkman index, mean BP, HbA1c, ALT, serum creatinine, triglycerides, total cholesterol, homocysteine, log homocysteine, FVC \% pred and FEV1 \% pred were not significantly different between the 523 male current smokers who were assessed in 2004-2006 and the 147 who performed follow-up spirometry in 2009 (data not shown). However, the mean age of these 147 subjects was significantly lower than that of the 523 subjects assessed in 2004-2006 (mean \pm SD $56.40 \pm 9.06$ versus $59.77 \pm 10.40$ years; $\mathrm{p}=0.0004$ ).

Histograms showing $\triangle \mathrm{FVC}$ and $\triangle \mathrm{FEV} 1$ per year are presented in online supplementary figure $\mathrm{S} 1$. As the lowest quintiles of decline in FVC (online supplementary fig. S1a) and FEV1 (online supplementary fig. S1b) were $-1.2 \%$ and $-2.5 \%$, respectively, the cut-off values for identifying subjects showing a rapid decline in pulmonary function were defined as annual rates of decline equivalent to, or greater than, these values. 28 subjects showed a decline in FVC and 29 showed a decline in FEV1 (table 4). Although FVC \% pred and FEV1 \% pred at the first visit did not differ between those who did, or did not, show a decline in FVC, the values at the second visit were significantly lower in those who did show a decline in FVC (table 4). FEV1/FVC at the first visit was significantly lower in those who showed a decline in FVC, compared with those who did not. However, this difference was not observed at the second visit. There were 
TABLE 2 Multivariate linear regression analyses of factors predictive for plasma homocysteine levels

Coefficient SE p-value

\begin{tabular}{lccc}
\hline Model 1 & & & \\
Age & 0.074 & 0.010 & $<0.0001$ \\
Male sex & 0.252 & 0.137 & 0.066 \\
BMI & -0.024 & 0.034 & 0.489 \\
Brinkman index & 0.001 & 0.000 & 0.004 \\
Mean BP & 0.031 & 0.010 & 0.001 \\
HbA1c & -0.319 & 0.157 & 0.041 \\
ALT & 0.016 & 0.008 & 0.039 \\
SCr & 6.517 & 0.598 & $<0.0001$ \\
Triglycerides & 0.004 & 0.002 & 0.011 \\
Total cholesterol & -0.014 & 0.003 & $<0.0001$ \\
FVC \% pred & -0.017 & 0.007 & 0.012 \\
Model 2 & & & $<0.0001$ \\
Age & 0.073 & 0.010 & 0.069 \\
Male sex & 0.250 & 0.137 & 0.488 \\
BMI & -0.024 & 0.035 & 0.007 \\
Brinkman index & 0.001 & 0.000 & 0.001 \\
Mean BP & 0.031 & 0.010 & 0.056 \\
HbA1c & -0.298 & 0.156 & 0.033 \\
ALT & 0.016 & 0.008 & $<0.0001$ \\
SCr & 6.548 & 0.598 & 0.009 \\
Triglycerides & 0.005 & 0.002 & $<0001$ \\
Total cholesterol & -0.014 & 0.003 & 0.048 \\
FEV1 \% pred & -0.012 & 0.006 & \\
\hline
\end{tabular}

BMI: body mass index; BP: blood pressure; $\mathrm{Hb}$ : haemoglobin; ALT: alanine aminotransferase; sCr: serum creatinine; FVC: forced vital capacity; \% pred: \% predicted; FEV1: forced expiratory volume in $1 \mathrm{~s}$.

no differences in age, BMI, Brinkman index, mean BP, HbA1c, ALT, triglycerides, total cholesterol and homocysteine levels between subjects who did, or did not, show a decline in FVC, the exception being serum creatinine values (table 4).

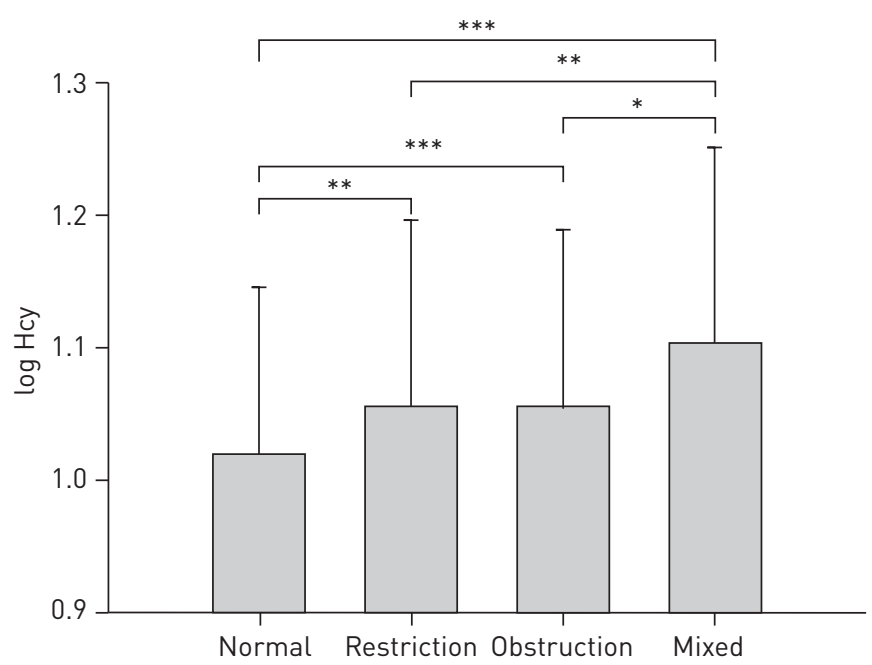

FIGURE 2 Plasma homocysteine (Hcy) levels according to the pattern of pulmonary function impairment. Subjects were classified into four groups according to their pulmonary function: normal (forced vital capacity (FVC) $\geqslant 80 \%$ predicted and forced expiratory volume in $1 \mathrm{~s}(\mathrm{FEV} 1) / \mathrm{FVC} \geqslant 0.7)(\mathrm{n}=2611)$; restriction $(\mathrm{FVC}<80 \%$ pred and FEV $1 / \mathrm{FVC} \geqslant 0.7)$ $(\mathrm{n}=190)$; obstruction (FVC $\geqslant 80 \%$ pred and FEV1/FVC $<0.7)(\mathrm{n}=261)$; and mixed $(\mathrm{FVC}<80 \%$ pred and FEV1/FVC $<0.7)(\mathrm{n}=72)$. Subjects with restrictive, obstructive or mixed impairment had higher Hcy levels than subjects with normal spirometry. In addition, subjects with mixed impairment had higher Hcy levels than subjects with either restrictive or obstructive impairment. Data on Hcy levels were not available for 123 subjects. Comparisons were performed by one-way ANOVA followed by the Student-Newman-Keuls test. ${ }^{*}: \mathrm{p}<0.05 ;{ }^{* *}: \mathrm{p}<0.01 ;{ }^{* *}: \mathrm{p}<0.001$. 
TABLE 3 Characteristics of male current smokers who performed a second pulmonary function test

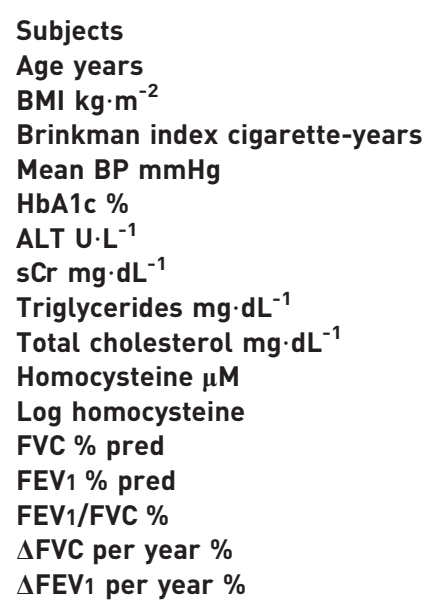

$$
\begin{gathered}
147 \\
56.40 \pm 9.06 \\
22.91 \pm 2.89 \\
751.7 \pm 413.8 \\
98.4 \pm 13.0 \\
5.12 \pm 0.54 \\
26.73 \pm 14.56 \\
0.747 \pm 0.113 \\
141.06 \pm 88.60 \\
194.01 \pm 29.84 \\
11.6(10.1-13.6) \\
1.07 \pm 0.12 \\
97.87 \pm 14.12 \\
96.57 \pm 16.58 \\
77.26 \pm 8.43 \\
-0.28 \pm 2.42 \\
-1.01 \pm 2.87
\end{gathered}
$$

\begin{tabular}{|c|c|c|c|c|c|c|}
\hline & FVC decline & No FVC decline & p-value & FEV1 decline & No FEV1 decline & p-value \\
\hline Subjects & 28 & 119 & & 29 & 118 & \\
\hline Age years & $61.79 \pm 10.42$ & $60.79 \pm 8.81$ & 0.651 & $63.55 \pm 9.50$ & $60.20 \pm 8.91$ & 0.063 \\
\hline $\begin{array}{l}\text { Brinkman index cigarette- } \\
\text { years }\end{array}$ & $737.6 \pm 409.8$ & $754.4 \pm 416.4$ & 0.866 & $863.3 \pm 387.8$ & $725.9 \pm 417.1$ & $0.028 *$ \\
\hline mean BP $\mathrm{mmHg}$ & $100.8 \pm 10.4$ & $97.8 \pm 13.5$ & 0.128 & $99.25 \pm 11.61$ & $98.19 \pm 13.38$ & 0.436 \\
\hline $\mathrm{sCr} \mathrm{mg} \cdot \mathrm{dL}^{-1}$ & $0.696 \pm 0.096$ & $0.759 \pm 0.114$ & $0.007^{*}$ & $0.728 \pm 0.107$ & $0.752 \pm 0.115$ & 0.3 \\
\hline Triglycerides $\mathrm{mg} \cdot \mathrm{dL}^{-1}$ & $148.57 \pm 97.66$ & $139.29 \pm 86.68$ & 0.632 & $132.58 \pm 67.66$ & $143.14 \pm 93.15$ & 0.851 \\
\hline Total cholesterol $\mathrm{mg} \cdot \mathrm{dL}^{-1}$ & $192.39 \pm 29.34$ & $194.40 \pm 30.07$ & 0.805 & $194.86 \pm 30.47$ & $193.80 \pm 29.81$ & 0.748 \\
\hline Homocysteine $\mu \mathrm{M}$ & $12.4(11.1-14.3)$ & $11.9(10.0-14.0)$ & 0.148 & $13.8(11.7-18.4)$ & $11.0(9.6-12.8)$ & $0.001^{*}$ \\
\hline $\begin{array}{l}\text { Log homocysteine } \\
\text { FVC \% pred }\end{array}$ & $1.09 \pm 0.11$ & $1.07 \pm 0.12$ & 0.148 & $1.13 \pm 0.11$ & $1.06 \pm 0.01$ & $0.001^{*}$ \\
\hline Second visit & $84.01 \pm 19.23$ & $98.73 \pm 16.35$ & $0.0001^{*}$ & $78.44 \pm 21.07$ & $100.23 \pm 14.01$ & $<0.0001^{*}$ \\
\hline \multicolumn{7}{|l|}{ FEV $1 /$ FVC $\%$} \\
\hline First visit & $74.27 \pm 7.68$ & $77.95 \pm 8.47$ & $0.039 *$ & $73.78 \pm 10.12$ & $78.11 \pm 7.77$ & 0.019* \\
\hline Second visit & $71.99 \pm 10.64$ & $73.27 \pm 9.02$ & 0.657 & $65.86 \pm 12.86$ & $74.79 \pm 7.28$ & $<0.0001 *$ \\
\hline
\end{tabular}

Data are presented as $n$, mean \pm SD or median (interquartile range). Data on homocysteine levels were not available for four subjects. Brinkman index data were not available for 19 subjects due to lack of information on precise smoking habits. BMI: body mass index; BP: blood pressure; Hb: haemoglobin; ALT: alanine aminotransferase; $\mathrm{sCr}$ : serum creatinine; FVC: forced vital capacity; \% pred: \% predicted; FEV1: forced expiratory volume in $1 \mathrm{~s} ; \Delta$ : change in.

TABLE 4 Characteristics of subjects who did, or did not, show a decline in pulmonary function

Data are presented as $n$, mean \pm SD or median (interquartile range), unless otherwise stated. Data on homocysteine levels were not available for four of the 147 subjects. Brinkman index data were not available for 19 subjects due to the lack of information on precise smoking habits. Differences in Brinkman index and homocysteine levels between subjects who did, or did not, show a decline in pulmonary function were evaluated by the MannWhitney U-test. Differences in all other variables were evaluated by t-test. BMI: body mass index; BP: blood pressure; Hb: haemoglobin; ALT: alanine aminotransferase; sCr: serum creatinine; FVC: forced vital capacity; \% pred: \% predicted; FEV1: forced expiratory volume in 1 s. 
TABLE 5 Multivariate logistic regression analyses of factors predictive for a decline in forced expiratory volume in $1 \mathrm{~s}$

\begin{tabular}{lll} 
& OR (95\% CI) & p-value \\
\hline Age (per 1-year increase) & $1.051(1.001-1.106)$ & 0.045 \\
Brinkman index (per 1-cigarette-year increase) & $1.121(0.960-1.318)$ & 0.150 \\
Homocysteine (per 1- $\mu$ M increase) & $1.100(1.010-1.206)$ & 0.030 \\
\hline
\end{tabular}

Data on homocysteine levels were not available for four of the 147 subjects. Brinkman index data were not available for 19 subjects due to lack of information on precise smoking habits.

FVC and FEV1 \% pred at the first visit did not differ between subjects who did, or did not, show a decline in FEV1 (table 4). However, at the second visit, FVC \% pred and FEV1 \% pred were significantly lower in subjects who did show a decline. FEV1/FVC at the first visit was significantly lower in subjects who showed a decline in FEV1, and this difference was greater at the second visit. Although age, BMI, mean BP, and HbA1c, ALT, serum creatinine, triglycerides and total cholesterol levels were not different, the Brinkman index and homocysteine levels were significantly higher in subjects showing a decline in FEV1 (table 4).

To investigate whether homocysteine levels were predictive for FEV1 decline, multivariate logistic regression analyses were performed. Homocysteine levels were significantly predictive for FEV1 decline (table 5).

Receiver operating characteristic curve analysis was performed to determine the optimum cut-off value of homocysteine for discriminating a decline in FEV1 among smokers. The optimum cut-off value for homocysteine was $11.1 \mu \mathrm{M}$, with an area under the curve of 0.7 , a sensitivity of 0.9 and a specificity of 0.5 (online supplementary fig. S2). Pulmonary function values for these subjects, relative to homocysteine levels (cut-off $11 \mu \mathrm{M}$ ), are shown in online supplementary table S2. At the first spirometric assessment, FVC \% pred, FEV1 \% pred and FEV1/FVC did not differ significantly between subjects with homocysteine levels $\leqslant 11$ or $>11 \mu \mathrm{M}$. At the second spirometric assessment, FVC \% pred and FEV1 \% pred were significantly lower in subjects with homocysteine levels $>11 \mu \mathrm{M}$ than in those with homocysteine levels $\leqslant 11 \mu \mathrm{M}$. The median annual rate of decline in FEV1 was significantly greater in subjects with homocysteine levels $>11 \mu \mathrm{M}$ than in those with homocysteine levels $\leqslant 11 \mu \mathrm{M}$.

\section{Discussion}

This study demonstrated that there was a significant correlation between spirometric parameters and homocysteine levels in subjects who participated in an annual health check. Furthermore, multiple linear regression analyses revealed that $\mathrm{FVC} \%$ pred and $\mathrm{FEV} 1 \%$ pred were significantly predictive for homocysteine levels, independent of age, sex, BMI, Brinkman index, mean BP, HbAlc, ALT, serum creatinine, triglyceride or total cholesterol levels. We can conclude that there are significant associations between spirometric measurements and homocysteine levels in the Japanese general population. In this cohort, subjects who showed a decline in FEV1 had higher homocysteine levels than those who did not. Logistic regression analysis demonstrated that homocysteine levels were a determinant of which subjects showed a decline in FEV1.

Homocysteine is an intermediate metabolite in the production of cysteine from methionine [30]. The factors contributing to hyperhomocysteinaemia are vitamin B deficiency [31], ageing [16], hypertension [20], renal dysfunction [24], diabetic nephropathy [21] and cigarette smoking [18, 19]. Multiple factors may regulate homocysteine levels, and, as such, determining the main cause(s) of hyperhomocysteinaemia in individual subjects is complex. Previous studies have demonstrated elevated homocysteine levels in COPD subjects, although the mechanism is still unknown $[14,15,32]$.

KAI et al. [14] demonstrated elevated homocysteine levels in COPD patients, and a positive, rather than an inverse, correlation between homocysteine levels and FEV1\% pred in a small number of COPD patients. This finding indicates that patients with mild airway obstruction may have higher homocysteine levels. In addition, in contrast to the inverse relationship between severity of airflow limitation and homocysteine levels, the latter were positively correlated with the annual rate of decline in FEV1 [14]. SEEMUNGAL et al. [15] also demonstrated elevated homocysteine levels in COPD patients. However, they demonstrated an inverse correlation between homocysteine levels and FEV1 \% pred in a small number of COPD patients. These previous studies showed contrasting results in terms of the correlation between severity of airflow obstruction and homocysteine levels. The present study clarifies this issue by demonstrating in a large population that poor lung function was associated with high homocysteine levels. In addition, high homocysteine levels were shown to be a predictor of FEV1 decline among male current smokers. 
FimOGNARI et al. [32] demonstrated that homocysteine levels were elevated in COPD patients and that low levels of folate and vitamin B12, as well as hyperglyceridaemia, were independent predictors of hyperhomocysteinaemia. The mechanism for the hyperhomocysteinaemia observed in COPD patients may simply be a decrease in folate and vitamin B12 or increased triglyceride levels. Unfortunately, we did not measure serum levels of folate and vitamin B12 in this study. As shown in table 2, triglyceride levels were associated with homocysteine levels, independent of other variables, although triglyceride levels did not differ between subjects who did or did not show a decline in FEV1 (table 4). A future study assessing the relationship between triglyceride levels and spirometric values in this population may be required.

The main cause of COPD is the long-term inhalation of cigarette smoke [1]. An imbalance of oxidants and antioxidants is one of the most important mechanisms in the development of COPD [1]. Pulmonary and systemic inflammation are recognised as important features of COPD, with regard to the development of airflow obstruction, and so are comorbidities such as atherosclerosis, weight loss and osteoporosis [33]. SEEMUNGAL et al. [15] also reported an association between homocysteine levels and C-reactive protein levels in COPD patients. Acute administration of homocysteine to rats is reported to result in elevated blood and brain concentrations of cytokines [34]. This suggests that hyperhomocysteinaemia may partly contribute to the systemic inflammation observed in COPD patients.

Hyperhomocysteinaemia is a well-known risk factor for cardiovascular disease [10-12]. Endothelial dysfunction induced by hyperhomocysteinaemia reportedly plays important roles in the development of atherosclerotic lesions [35], and homocysteine is associated with apoptosis in endothelial progenitor cells that play important roles in vascular endothelial homeostasis [36]. Therefore, it is hypothesised that lowering homocysteine levels with the use of vitamin B may potentially attenuate the development of atherosclerosis. However, some randomised control trials reveal that treating hyperhomocysteinaemia with B vitamins does not decrease vascular events, suggesting that hyperhomocysteinaemia is only a marker of overall vascular risk [37-39].

Cardiovascular disease is a frequent comorbidity in patients with COPD [40]. Accumulating evidence suggests that pulmonary endothelial dysfunction is involved in the pathogenesis of COPD [41-43]. We have previously shown that the rate of washout of ${ }^{123} \mathrm{I}$-metaiodobenzylguanidine, a specific scintigraphic molecule that enables measurement of endothelial function in vivo, was decreased in patients with COPD [41]. Many risk factors, including hypertension, hyperglycaemia, hyperglyceridaemia, hypercholesterolaemia and smoking, contribute to the pathogenesis and development of atherosclerosis. Therefore, even if subjects receive therapy with vitamin B to lower homocysteine levels, cardiovascular events may not be reduced, due to the significant effects of other risk factors such as hypertension, hyperglycaemia, hyperglyceridaemia, hypercholesterolaemia and smoking. In contrast to atherosclerosis, the risk factors for the development of COPD are simple, and cigarette smoking is the most important risk factor. Although the results of trials of the effects of vitamin B on cardiovascular events were negative [37-39], therapy to lower homocysteine levels may protect the lungs from injury induced by cigarette smoke. Clinical trials investigating the potential of homocysteine-lowering therapy for COPD patients may be required. In addition, measuring homocysteine levels may be useful in identifying patients with COPD, who are at higher risk of vascular events, and a more rapid decline in lung function.

As mentioned previously, homocysteine levels are influenced by various other factors, including age, sex, blood pressure, blood glucose levels and renal function. Therefore, careful consideration of these other factors is required to assess the relationship between pulmonary function and homocysteine levels. In the present study, multiple linear regression analyses were performed to control for the influence of factors other than homocysteine levels on pulmonary function. OzKAn et al. [44] previously demonstrated that serum homocysteine levels were significantly higher in patients with severe sleep apnoea syndrome than in controls, suggesting that tissue hypoxia may be associated with the level of serum homocysteine. However, arterial oxygen tension and saturation were not measured in this population, and this was a limitation of our study.

A further limitation was sampling bias. Takahata town has a resident population of 15222 adults, aged $\geqslant 40$ years (7109 males and 8113 females). Data for a total of 3257 enrolled subjects (1502 males and 1755 females) were entered into the final statistical analysis. Among the 1502 male subjects, there were 523 active smokers. However, follow-up spirometry in 2009 was only performed for 147 subjects who had continued to smoke. The remaining subjects were lost to follow-up or quit smoking before follow-up. The number of subjects who were followed-up was not large; however, these 147 subjects appeared to be representative of the 523 male active smokers from the first assessment. The only significant difference between these groups was age (see the Results section). In addition, workers in public institutions and many companies did not participate in this research programme for reasons described previously [25]. Differences in type of 
employment, socioeconomic status or lifestyle, including vitamin B intake, between participants and nonparticipants may have influenced the results.

In the present study, all subjects were Japanese, and those assessed for a decline in FEV1 were 147 male active smokers. The subject number may not have been sufficient to obtain accurate cut-off levels of homocysteine for discriminating those who showed a decline in FEV1. In addition, this cut-off (homocysteine $>11 \mu \mathrm{M}$ ) may not be applicable to other ethnic groups, or to females. Further investigation is required to determine the cut-off values of homocysteine according to ethnicity and sex.

In conclusion, homocysteine levels were significantly associated with FVC \% pred and FEV1 \% pred in the Japanese general population, and were identified as a significant predictive factor for decline in FEV1 among male smokers. Measuring homocysteine levels in smokers may facilitate smoking cessation by providing an indication of individual susceptibility to cigarette smoke and its effect on decline in lung function.

\section{Acknowledgements}

We thank Taiko Aita, Emiko Nakamura and Eiji Tsuchida (Yamagata University, Yamagata, Japan), Michiko Nishiwaki (Yamagata City Hospital, Saiseikan, Japan), Toshihiro Wada (Yamagata City Hospital, Saiseikan, Japan), Jun-Ichi Machiya (Nihonkai General Hospital, Yamagata, Japan), Noriyuki Hirama (National Hospital Organisation Yamagata National Hospital, Yamagata, Japan), Noriaki Takabatake (Tohoku Central Hospital, Yamagata, Japan) and Makoto Sata (National Cerebral and Cardiovascular Centre, Osaka, Japan) for their contribution and excellent assistance. We also thank Toshiro Tango (Centre for Medical Statistics, Tokyo, Japan) and Sharon Forsyth (BioMedical Editing International, WA, Australia) for evaluating the statistical analysis and English language editing, respectively.

\section{References}

1 Global Initiative for Chronic Obstructive Lung Disease. Global Strategy for the Diagnosis, Management and Prevention of Chronic Obstructive Pulmonary Disease, 2013. www.goldcopd.org/uploads/users/files/GOLD_Report_ 2013_Feb20.pdf Date last accessed: May 10, 2013. Date last updated: February 2013.

2 Osaka D, Shibata Y, Abe S, et al. Relationship between habit of cigarette smoking and airflow limitation in healthy Japanese individuals: the Takahata study. Intern Med 2010; 49: 1489-1499.

3 Iwamoto $\mathrm{H}$, Yokoyama A, Kitahara $\mathrm{Y}$, et al. Airflow limitation in smokers is associated with subclinical atherosclerosis. Am J Respir Crit Care Med 2009; 179: 35-40.

4 Maclay JD, McAllister DA, Macnee W. Cardiovascular risk in chronic obstructive pulmonary disease. Respirology 2007; 12: 634-641.

5 Maggi S, Siviero P, Gonnelli S, et al. Osteoporosis risk in patients with chronic obstructive pulmonary disease: the EOLO study. J Clin Densitom 2009; 12: 345-352.

6 Mannino DM, Thorn D, Swensen A, et al. Prevalence and outcomes of diabetes, hypertension and cardiovascular disease in COPD. Eur Respir J 2008; 32: 962-969.

7 Takabatake N, Sata M, Inoue S, et al. A novel polymorphism in secretory phospholipase A2-IID is associated with body weight loss in chronic obstructive pulmonary disease. Am J Respir Crit Care Med 2005; 172: 1097-1104.

8 Wouters EF, Creutzberg EC, Schols AM. Systemic effects in COPD. Chest 2002; 121: 127S-130S.

9 Yao H, Rahman I. Current concepts on the role of inflammation in COPD and lung cancer. Curr Opin Pharmacol 2009; 9: 375-383.

10 Glueck CJ, Shaw P, Lang JE, et al. Evidence that homocysteine is an independent risk factor for atherosclerosis in hyperlipidemic patients. Am J Cardiol 1995; 75: 132-136.

11 Hladovec J. Homocysteine theory of atherosclerosis. Sb Lek 1995; 96: 225-230.

12 Taylor LM Jr, Porter JM. Elevated plasma homocysteine as a risk factor for atherosclerosis. Semin Vasc Surg 1993; 6 : 36-45.

13 Andersson A, Ankerst J, Lindgren A, et al. Hyperhomocysteinemia and changed plasma thiol redox status in chronic obstructive pulmonary disease. Clin Chem Lab Med 2001; 39: 229-233.

14 Kai S, Nomura A, Morishima Y, et al. The effect of smoking-related hyperhomocysteinemia on spirometric declines in chronic obstructive pulmonary disease in elderly Japanese. Arch Gerontol Geriatr 2006; 42: 117-124.

15 Seemungal TA, Lun JC, Davis G, et al. Plasma homocysteine is elevated in COPD patients and is related to COPD severity. Int J Chron Obstruct Pulmon Dis 2007; 2: 313-321.

16 Powers RW, Majors AK, Lykins DL, et al. Plasma homocysteine and malondialdehyde are correlated in an age- and gender-specific manner. Metabolism 2002; 51: 1433-1438.

17 Guzelmeric K, Alkan N, Pirimoglu M, et al. Chronic inflammation and elevated homocysteine levels are associated with increased body mass index in women with polycystic ovary syndrome. Gynecol Endocrinol 2007; 23: 505-510.

18 Stein $\mathrm{JH}$, Bushara $\mathrm{M}$, Bushara $\mathrm{K}$, et al. Smoking cessation, but not smoking reduction, reduces plasma homocysteine levels. Clin Cardiol 2002; 25: 23-26.

19 Zamboni M, Di Francesco V, Zoico E, et al. Homocysteine and life-style in the elderly. Aging 2001; 13: 437-442.

20 Kennedy BP, Farag NH, Ziegler MG, et al. Relationship of systolic blood pressure with plasma homocysteine: importance of smoking status. J Hypertens 2003; 21: 1307-1312.

21 Audelin MC, Genest J Jr. Homocysteine and cardiovascular disease in diabetes mellitus. Atherosclerosis 2001; 159: 497-511.

22 Huang L, Song XM, Zhu WL, et al. Plasma homocysteine and gene polymorphisms associated with the risk of hyperlipidemia in northern Chinese subjects. Biomed Environ Sci 2008; 21: 514-520.

23 Remkova A, Remko M. Homocysteine and endothelial markers are increased in patients with chronic liver diseases. Eur J Intern Med 2009; 20: 482-486.

24 Gale CR, Ashurst H, Phillips NJ, et al. Renal function, plasma homocysteine and carotid atherosclerosis in elderly people. Atherosclerosis 2001; 154: 141-146. 

in a health check: the Takahata study. Int J Med Sci 2011; 8: 470-478.

26 Kishi H, Shibata Y, Osaka D, et al. FEV6 and FEV1/FEV6 in Japanese participants of the community-based annual health check: the Takahata study. Intern Med 2011; 50: 87-93.

27 Nemoto T, Shibata Y, Osaka D, et al. Impact of cigarette smoking on maximal expiratory flows in a general population: the Takahata study. Intern Med 2011; 50: 2547-2555.

28 Kellogg MD, Parker R, Ricupero A, et al. Evaluation of an enzymatic homocysteine assay for the Hitachi series chemistry analyzer. Clin Chim Acta 2005; 354: 117-122.

29 The Committee of Pulmonary Physiology. Guidelines for Pulmonary Function Tests: Spirometry, Flow-volume Curve, Diffusion Capacity of the Lung. Tokyo, JRS, 2004.

30 D'Angelo A, Selhub J. Homocysteine and thrombotic disease. Blood 1997; 90: 1-11.

31 Ubbink JB. Vitamin nutrition status and homocysteine: an atherogenic risk factor. Nutr Rev 1994; 52: 383-387.

32 Fimognari FL, Loffredo L, Di Simone S, et al. Hyperhomocysteinaemia and poor vitamin B status in chronic obstructive pulmonary disease. Nutr Metab Cardiovasc Dis 2009; 19: 654-659.

33 Fabbri LM, Rabe KF. From COPD to chronic systemic inflammatory syndrome? Lancet 2007; 370: 797-799.

34 da Cunha AA, Ferreira AG, Wyse AT. Increased inflammatory markers in brain and blood of rats subjected to acute homocysteine administration. Metab Brain Dis 2010; 25: 199-206.

35 Lentz SR, Rodionov RN, Dayal S. Hyperhomocysteinemia, endothelial dysfunction, and cardiovascular risk: the potential role of ADMA. Atheroscler Suppl 2003; 4: 61-65.

36 Bao XM, Wu CF, Lu GP. Atorvastatin inhibits homocysteine-induced oxidative stress and apoptosis in endothelial progenitor cells involving Nox4 and p38MAPK. Atherosclerosis 2010; 210: 114-121.

37 Potter K, Hankey GJ, Green DJ, et al. The effect of long-term homocysteine-lowering on carotid intima-media thickness and flow-mediated vasodilation in stroke patients: a randomized controlled trial and meta-analysis. BMC Cardiovasc Disord 2008; 8: 24.

38 Ebbing M, Bleie O, Ueland PM, et al. Mortality and cardiovascular events in patients treated with homocysteinelowering B vitamins after coronary angiography: a randomized controlled trial. JAMA 2008; 300: 795-804.

39 Albert CM, Cook NR, Gaziano JM, et al. Effect of folic acid and B vitamins on risk of cardiovascular events and total mortality among women at high risk for cardiovascular disease: a randomized trial. JAMA 2008; 299 : $2027-2036$.

40 Rodriguez-Roisin R, Soriano JB. Chronic obstructive pulmonary disease with lung cancer and/or cardiovascular disease. Proc Am Thorac Soc 2008; 5: 842-847.

41 Arao T, Takabatake N, Sata M, et al. In vivo evidence of endothelial injury in chronic obstructive pulmonary disease by lung scintigraphic assessment of ${ }^{123}$ I-metaiodobenzylguanidine. J Nucl Med 2003; 44: 1747-1754.

42 Kasahara Y, Tuder RM, Cool CD, et al. Endothelial cell death and decreased expression of vascular endothelial growth factor and vascular endothelial growth factor receptor 2 in emphysema. Am J Respir Crit Care Med 2001; 163: 737-744.

43 Kasahara Y, Tuder RM, Taraseviciene-Stewart L, et al. Inhibition of VEGF receptors causes lung cell apoptosis and emphysema. J Clin Invest 2000; 106: 1311-1319.

44 Ozkan Y, Firat H, Simsek B, et al. Circulating nitric oxide (NO), asymmetric dimethylarginine (ADMA), homocysteine, and oxidative status in obstructive sleep apnea-hypopnea syndrome (OSAHS). Sleep Breath 2008; 12: $149-154$. 